\title{
Inquérito entomológico e infectividade durante epidemia de malária no município de Anajás, Estado do Pará
}

\author{
Entomological survey and infectivity during malaria outbreak \\ in the Anajás municipality, Pará State
}

\author{
Roseli La Corte dos Santos ${ }^{1,2}$, Izis Mônica Carvalho Sucupira ${ }^{3}$, Raimundo Nonato \\ da Luz Lacerda ${ }^{3}$, Arnaldo da Silva Fayal ${ }^{4}$ e Marinete Marins Póvoa ${ }^{3}$
}

\begin{abstract}
RESUM0
Inquérito entomológico transversal foi conduzido na área urbana no Município de Anajás/PA, para verificar o risco de transmissão malárica. Embora a densidade de Anopheles tenha sido baixa, a taxa de infectividade de $6 \%$ e 0 encontro de espécimes positivos em todos os bairros indicam alto risco de contrair a doença.
\end{abstract}

Palavras-chaves: Anopheles darlingi. Infectividade. Malária. Anajás. Amazônia.

\begin{abstract}
An entomological survey was conducted in the urban area of Anajás municipality, Pará State, in order to verify the risk of malaria transmission. Although the density of Anopheles species was low, the anopheline infectivity rate of $6 \%$ demonstrated there is a high risk of infection by the malaria parasites.
\end{abstract}

Key-words: Anopheles darlingi. Infectivity. Malaria. Anajas. Amazon.

Entre os Estados que constituem a Amazônia brasileira, 0 Pará é um dos que apresenta maior morbidade por malária. Nos anos de 2001 e 2002, esse estado contribuiu com 48\% e $53,5 \%$ do total de casos registrados na Amazônia Legal,

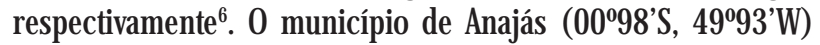
representa área de alto risco para malária, com incidência parasitária anual em torno de 450 casos por mil habitantes. Em anos epidêmicos, como 2001 e 2002, essa incidência atingiu 0 patamar de 884 e 797 casos por mil habitantes, respectivamente 6 .

A principal atividade econômica do município é a extração de palmito de açaí. Anotificação dos casos de malária é realizada na cidade de Anajás e por isso formou-se 0 conceito de que 0 risco de transmissão seria baixo na área urbana e que os casos lá notificados seriam de pessoas envolvidas nas atividades extrativistas. 0 presente estudo foi realizado com o objetivo de verificar o risco de transmissão na área urbana do município de Anajás, utilizando informações entomológicas.
0 estudo foi realizado como parte das atividades da divisão de Entomologia da Secretaria de Estado da Saúde do Pará e condurido durante 0 mês de março de 2002 na área urbana do município. Mosquitos foram capturados com uso de aspirador manual no momento do repasto sangǘneo, em ambiente peri domiciliar. Capturas foram realizadas das 18 às 21 horas, em 8 pontos por dia, durante 4 dias seguidos. 0 total de 19 pontos foram distribuídos ao longo da cidade. Foram realizadas capturas em mais dois dias, com 12 horas de duração, das 18 às 6 horas da manhã, em 3 pontos diferentes, os quais apresentaram maior rendimento nas capturas anteriores.

Mosquitos do gênero Anopheles foram identificados e 30\% das fêmeas de Anopheles darlingi, tiveram suas glândulas salivares, estômagos e ovários dissecados. As cabeças e tórax de todo material, dissecado ou não, foram submetidos a testes de imunoadsorção enzimática (ELISA). Outros Culicidae foram enviados à Faculdade de Saúde Pública da Universidade de São Paulo para identificação.

\footnotetext{
1. Secretaria de Vigilância em Saúde, Brasília, DF. 2. Núcleo de Pesquisa Taxonômica e Sistemática em Entomologia Médica (NUPTEN) da Faculdade de Saúde Pública da Universidade de São Paulo, São Paulo, SP. 3. Instituto Evandro Chagas da Secretaria de Vigilância em Saúde, Belém, PA. 4. Divisão de Entomologia da Secretaria Especial de Proteção Social do Estado do Pará, Belém, PA.

Apoio financeiro: Secretaria de Vigilância em Saúde ( SVS) , Instituto Evandro Chagas/SVS e Secretaria de Saúde do Estado do Pará.

Endereço para correspondência: Dra. Roseli La Corte dos Santos. SEPN 511 Bloco C - 3o andar, 70750-593 Brasília, DF, Brasil

Tel.: 5561 448-8772

E-mail: lacorte@saude.gov.br, rlacorte@usp.br

Recebido para publicação em 10/11/2003

Aceito em 30/11/2004
} 
Foram capturados 2.864 mosquitos (Tabela 1) no período do estudo. Mosquitos do gênero Culex foram os mais abundantes, representando $82,7 \%$ do total. 0 segundo gênero mais abundante foi Anopheles (15,8\%), sendo que An. darlingi apresentou maior número de espécimens.

Tabela 1 - Distribuição de mosquitos adultos coletados por espécie, Anajás, PA, março de 2002.

\begin{tabular}{lrr}
\hline & \multicolumn{2}{c}{ Quantidade } \\
\cline { 2 - 3 } Espécie & $\mathrm{n}^{\underline{0}}$ & $\%$ \\
\hline Culex (Melanoconion) delpontei & 1.620 & 59,9 \\
Culex (Culex) quinquefasciatus & 469 & 17,4 \\
Culex (Melanoconion) sp danificado & 81 & 3,0 \\
Culex (Melanoconion) theobaldi & 28 & 1,0 \\
Culex (Culex) Grupo Coronator & 24 & 0,9 \\
Culex (Culex) usquatus & 8 & 0,3 \\
Culex (Melanoconion) sp. Seção Melanoconion & 4 & 0,2 \\
Culex (Melanoconion) ocossa & 1 & 0,0 \\
Total parcial & 2.235 & 82,7 \\
Anopheles darlingi & 381 & 14,1 \\
Anopheles oswaldoi & 22 & 0,8 \\
Anopheles nuneztovari & 18 & 0,7 \\
Anopheles triannulatus & 6 & 0,2 \\
Anopheles albitarsis & 1 & 0,0 \\
Anopheles intermedius & 1 & 0,0 \\
Total parcial & 429 & 15,8 \\
Coquilletidia venezuelensis & 18 & 0,7 \\
Ochlerotatus scapularis & 16 & 0,6 \\
Uranotaenia geometrica & 4 & 0,2 \\
Total parcial & 38 & 1,5 \\
Total identificado & 2.702 & 100,0 \\
Danificados & 162 & \\
\hline Total capturado & 2.864 & \\
\hline & \multicolumn{3}{c}{}
\end{tabular}

As capturas de 12 horas, embora com menor número de horas trabalhadas (144) foi mais produtiva que as capturas de 3 horas (180). 0 rendimento médio nas capturas de 12 horas foi de 1,9 mosquito/homem/hora, enquanto que nas capturas de três horas esse valor foi de 0,8 mosquito/homem/hora. 0 pico de atividade de An. darlingi concentrou-se entre 21 e 24 horas (Figura 1).

Do total de anofelinos capturados, 417 foram analisados pelo teste de ELISA sendo que 25 apresentaram-se positivos, taxa de infectividade igual a 6\% (Tabela 2). Em todos os bairros da cidade foram encontrados mosquitos positivos. Entre as 116 fêmeas de An. darlingi dissecadas, 3 apresentavam grande quantidade de esporozoítos em suas glândulas salivares e oocistos nos estômagos, sendo a taxa de infectividade por essa técnica igual

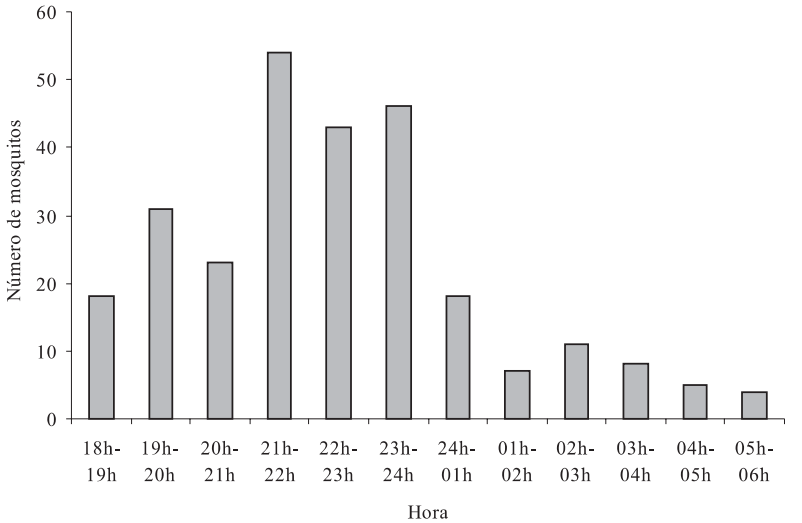

Figura 1 - Atividade horária de Anopheles darlingi coletados em isca humana, Anajás, PA, março de 2002.

a 2,7\%. Dos ovários dissecados, 55 (47,4\%) apresentavam dilações.

Condições favoráveis à transmissão foram detectadas na área urbana do município de Anajás. Embora a densidade de An darlingi não tenha sido elevada, essa espécie esteve presente em todos os bairros da cidade. Todavia, em que pese a densidade de mosquitos ter sido baixa, 1,9 mosquito/homem/hora, isso considerando a técnica e os pontos mais produtivos, a taxa de infectividade de $6 \%$ foi muito superior às verificadas em outras áreas endêmicas ${ }^{2} 3$ 5 0 encontro de 3 mosquitos infectados, detectados pela técnica de dissecção de glândulas salivares confirma a alta infectividade da área, uma vez que essa técnica é menos sensível que a de ELISA e, em geral, mesmo em áreas endêmicas, número muito maior de mosquitos necessita ser dissecado para que se detecte infecção natural. Os resultados indicam também a circulação de Plasmodium malariae, devido à detecção dessa espécie em dois exemplares de mosquitos, sendo um An darlingi e o outro An oswaldoi, fato já observado em outras áreas da Amazônia' ${ }^{1}$. Vale ressaltar a possibilidade de participação secundária de outros Anopheles na transmissão, principalmente An. oswaldoi, considerando-se que dois dos 20 mosquitos testados estavam positivos.

Com relação à atividade horária, notou-se maior atividade entre 21 e 24 horas e não crepuscular como já registrado em outras áreas. ${ }^{4}$. Além disso, An. darlingi foi capturado durante todas as horas da noite, comportamento já descrito por diversos autores $^{78}$. Tadei et al ${ }^{8}$ estudando populações de An. darlingi da região da usina hidrelétrica de Tucuruí no Pará, verificaram picos não crepusculares, entre 20 e 22 horas, e atividade hematofágica durante toda a noite, como no presente estudo. Todavia as informações aqui registradas foram pontuais e,

Tabela 2 - Distribuição de mosquitos positivos para plasmódio, segundo espécie, Anajás, PA, março de 2002.

\begin{tabular}{lccccc}
\hline Espécie & $\begin{array}{c}\text { Plasmodium } \\
\text { vivax }\end{array}$ & $\begin{array}{c}\text { Plasmodium vivax } \\
\text { variante VK247 }\end{array}$ & $\begin{array}{c}\text { Plasmodium } \\
\text { falciparum }\end{array}$ & $\begin{array}{c}\text { Plasmodium } \\
\text { malariae }\end{array}$ & $\begin{array}{c}\text { Total } \\
\text { TMI/TMT }(\%)\end{array}$ \\
\hline Anopheles darlingi & 10 & 0 & 11 & 1 & $22 / 372(5,9)$ \\
Anopheles oswaldoi & 0 & 1 & 0 & 1 & $2 / 20(10,0)$ \\
Anopheles nuneztovari & 1 & 0 & 0 & 0 & $1 / 18(5,5)$ \\
\hline Total & 11 & 1 & 11 & 2 & $25 / 417 \mathrm{a}(6,0)$ \\
\hline
\end{tabular}

* TMI = total de mosquitos infectados

TMT $=$ total de mosquitos testados

a Total incluindo espécies negativas 
considerando a plasticidade intrapopulacional que pode ocorrer nas populações de An darlingi ${ }^{9}$, seria precipitado concluir sobre a atividade horária dessa espécie em Anajás, todavia, diante do encontrado, é recomendável atentar a este fato por ocasião do planejamento de ações para o controle de mosquitos adultos.

A taxa de paridade de $47,4 \%$ também revela que quase metade da população de mosquitos em Anajás já havia realizado ovipostura, o que significa mais um fator favorável à transmissão, uma vez que as fêmeas estão sobrevivendo tempo suficiente para a realização do ciclo extrínseco do parasita, fato confirmado pela alta (6\%) taxa de infectividade encontrada.

Com base nas informações entomológicas obtidas, embora uma pessoa receba poucas picadas por noite na área urbana de Anajás, a população deste município está sob alto risco de contrair a doença, em qualquer bairro da cidade, tendo em vista a elevada taxa de infectividade verificada.

\section{AGRADECIMENTOS}

Os autores agradecem a A. Fernandes pela identificação dos culicíneos, a R. Lessa pela realização do teste ELISA e à equipe de campo: D. Galiza Primo, A.P.H. Diniz, J.M. Nascimento, R.N. Almeida, F.G. Santos Filho, G.G. Barbosa, R.B. Baia, A.0. Bandeira, P. Conceição Júnior, M. Garcia e R.J.F Silva.

\section{REFERÊNCIAS BIBLIOGRÁFICAS}

1. Arruda M, Nardin EH, Nussenzweig RS, Cochrane AH. Sero-epidemiological studies of malaria in Indian tribes and monkeys of the Amazon Basin of Brazil. American Journal of Tropical Medicine and Hygiene 41:379-385, 1989.

2. Branquinho MS, Taipe-Lagos CB, Rocha R, Natal D, Barata JMS, Cochrane $\mathrm{AH}$, Nardin E, Nussenzweig RS, Kloetzel JK. Anophelines in the State of Acre, Brazil, infected with Plasmodium falciparum, Plasmodium vivax, the variant Plasmodium vivax VK247 and Plasmodium malariae. Transactions of the Royal Society of Medicine and Hygiene 87:391-394, 1993.

3. Oliveira-Ferreira J, Lourenço-de-Oliveira R, Teva A, Deane LM, Daniel-Ribeiro CT. Natural malaria infections in anophelines in Rondonia State, Brazilian Amazon. American Journal of Tropical Medicine and Hygiene 43:6-10, 1990.

4. Póvoa MM, Silva ANM, Santos CCB, Segura MNO, Machado RLD. Malaria transmission. Ciência e Cultura 52:208-212, 2000.

5. Póvoa MM, Wirtz RA, Lacerda RNL, Miles MA, Warhurst D. Malaria vectors in the municipality of Serra do Navio, State of Amapá, Amazon region, Brazil. Memórias do Instituto Oswaldo Cruz 96:179-184, 2001.

6. Secretaria de Vigilância em Saúde. SIVEP (2000-2002) Sistema de informação em malária. Brasília, 2003.

7. Silva-Vasconcelos A, Kató MYN, Mourão EM, Souza RTL, Lacerda RNL, Sibajev A, Tsouris P, Póvoa MM, Momen H, Rosa-Freitas MG. Biting indices, host-seeking activity and natural infection rates of anopheline species in Boa Vista, Roraima, Brazil from 1996 to 1998. Memórias do Instituto Oswaldo Cruz 97:151-161, 2002.

8. Tadei WP, Mascarenhas BM, Podestá MG. Biologia de anofelinos amazônicos. VIII. Conhecimentos sobre a distribuição de espécies de Anopheles na região de Tucuruí-Marabá ( Pará). Acta Amazonica 13:103-140, 1983.

9. Voorham J. Intra-population plasticity of Anopheles darlingi's ( Diptera: Culicidae) biting activity patterns in the State of Amapá, Brazil. Revista de Saúde Pública 36:75-80, 2002. 